\title{
Terahertz spectroscopy for diabetes diagnostics
}

\author{
O.P. Cherkasova ${ }^{1}$, M.M. Nazarov ${ }^{2}$, A.P. Shkurinov ${ }^{3,4}$ \\ ${ }^{1}$ Institute of Laser Physics of SB RAS, Novosibirsk, Russia, o.p.cherkasova@gmail.com \\ ${ }^{2}$ Kurchatov Institute National Research Center, Moscow, Russia \\ ${ }^{3}$ Crystallography and Photonics Federal Research Center RAS, Moscow, Russia \\ ${ }^{4}$ Lomonosov Moscow State University, Moscow, 119991, Russia
}

\section{Introduction}

Diabetes mellitus is a chronic disease caused by inherited and/or acquired deficiency in production of insulin by the pancreas, or by the ineffectiveness of the produced insulin. Such a deficiency results in increased concentrations of glucose in the blood, which in turn damage many of the body's systems, in particular the blood vessels and nerves. The development of diabetes mellitus leads to violation of carbohydrate, protein, and lipid exchange and is accompanied by a significant increase in the content of glucose, corticosteroid hormones, and some other metabolites in blood. The development of new rapid diagnostic methods for diabetes and its complications is an urgent problem.

Terahertz (THz) radiation has a number of useful features for medical applications. It is sensitive to water concentration and state, it does not ionize biological objects [1] and passes through the skin [2]. A distinctive feature of $\mathrm{THz}$ time domain spectroscopy is the possibility of measuring directly the refractive index and absorption coefficient, and hence complex permittivity spectrum of the sample in a single scan and in a broad frequency range [3]. This circumstance makes it possible to obtain a detailed spectral characteristic of a sample during one measurement, due to which rapid and even remote diagnostics is foreseen.

\section{Experimental}

Our $\mathrm{THz}$ time-domain apparatus was described previously $[4,5]$. We used the radiation of a Ti:sapphire laser with a wavelength of $790 \mathrm{~nm}$, a pulse duration of $90 \mathrm{fs}$ with $1 \mathrm{Wt}$ average power. For $\mathrm{THz}$ emission, the semiconductor (LT-GaAs) surface was illuminated by laser beam. For $\mathrm{THz}$ detection the electro-optical ZnTe crystal of $1 \mathrm{~mm}$ thickness was probed by a small, delayed fraction of laser beam. We have used both transmission (for blood plasma) and attenuated total internal reflection (ATR) configurations (for blood plasma and skin). The spectral range of reliable measurements was between 0.05 and $3.2 \mathrm{THz}$. We analyzed blood plasma of a rat with experimental diabetes. In additional the ATR spectra of human skin on the palm of the hand in vivo were measured at a normal blood glucose concentration (4.2-5.9 mM) and 45 and 90 minutes later after drinking the glucose solution ( $75 \mathrm{~g}$ per $100 \mathrm{ml}$ of water). This is the standard oral glucose tolerance test, which demonstrates an initial spike and rapidly returns to normal levels of blood glucose concentration.

\section{Results}

$\mathrm{THz}$ spectroscopy of biological samples mainly probes water strong absorption and dispersion in $\mathrm{THz}$ ranges. It is known that relaxation time of water molecules involved in hydration of biomolecules are different from those of characterizing free, unbound water molecules, which is reflected in the low-frequency absorption and refraction spectra of biological samples (tissues, blood plasma) [4]. We have previously shown the insertion of glucose into water leads only to an increase of relaxation time $\tau_{1}$ of the slow Debye process of this solution. The increasing of protein albumin concentration in the solution results in a decrease of the amplitude $\Delta \varepsilon_{1}$ of the slow Debye relaxation process [5]. This explains observed sensitivity of $\mathrm{THz}$ skin reflection spectra and blood plasma properties.

The figure $1 \mathrm{a}$ ) shows the averaged absorption coefficient difference for healthy and rat with alloxane diabetes. That is the difference between distilled water and studied solution.

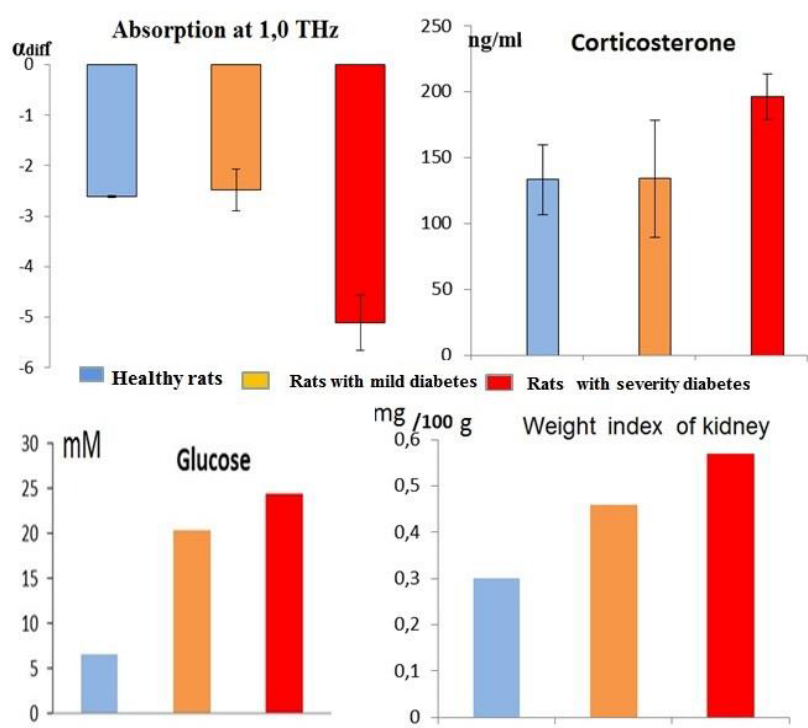

Fig. 1. The absorption coefficient differences at $1 \mathrm{THz}$, corticosterone and glucose concentration in blood plasma, and weight index of kidney of healthy (blue), mild diabetics (orange) and severity diabetics (red) rats

The absorption coefficient differs in group of rats with severity diabetes by a factor of more than two from the corresponding value in other groups. This fact indicates that some components secreted into the blood plasma under experimental diabetes complications may contribute to the total absorption. The rats with severity diabetes differed by a reliably high level of corticosteroid hormones in blood and adrenal glands, a large weight index of kidney, and a higher level of glucose in blood in comparison with the healthy and mild diabetics rats [6]. The decrease in the amplitudes of absorption spectra of blood plasma of rats with severity diabetes can be consid- 
ered as an integral estimate of significant biochemical disturbances in Diabetes.

Assuming that the observed spectral changes are due to changes in the state of water in blood plasma, we have selected one of the parameters of the Debye model aqueous solution $-\Delta \varepsilon_{l} / \tau_{1}$, leading to the spectral features observed in the experiment. This change in the response of bound water can be the reason of the observed changes in our experiments at increasing glucose concentration in blood. We have demonstrated that when the concentration of glucose in blood rises to $24 \mathrm{mM}$ (in rats with severity diabetes), $\Delta \varepsilon_{1} / \tau_{\mathrm{D}}$ ratio decreases 1.2 times.

The correlation of the ATR amplitude of human skin and glucose concentration in blood is demonstrated in figure 2 . In this case we use ATR amplitude $R_{\text {int }}$ integrated over the used frequency range, and time shift $\Delta \mathrm{T}$ [7]. It can be seen that the variations of the optical characteristics of human skin correlate with the changes in blood glucose level.
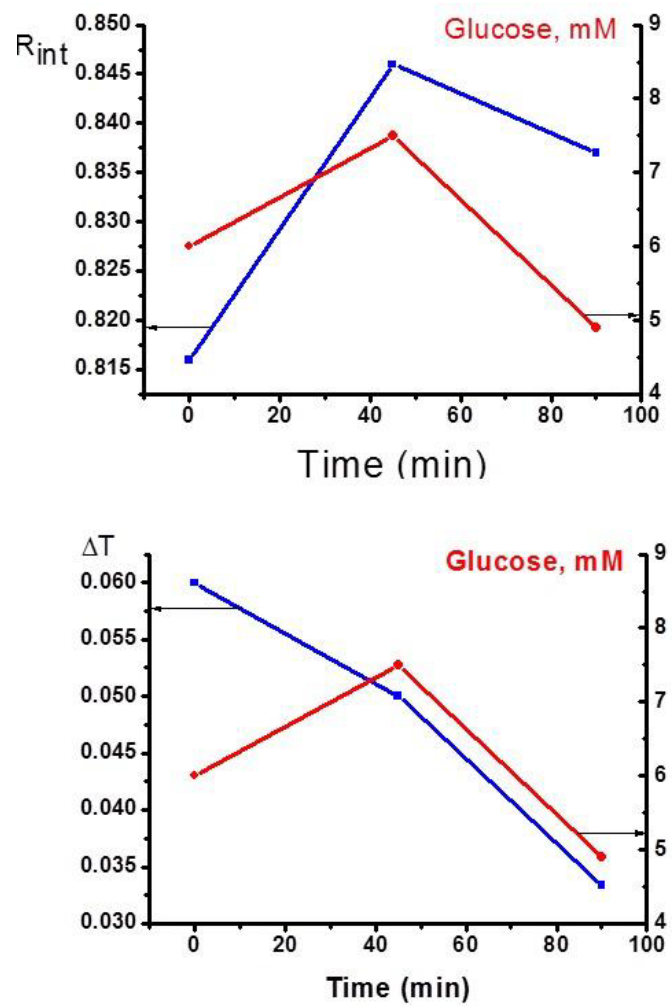

Fig. 2. The ATR amplitude $R_{\text {int }}$ and time shift $\Delta T$ of human skin and glucose concentration in blood $(\mathrm{mM})$ versus time ( $\mathrm{min})$ after glucose intake

The change in the ATR amplitude and phase of skin are determined only by variations of the slow Debye process [7]. When we observe changes of the ATR spectra after ingestion of glucose solution we see the effect that has a high level of blood glucose concentration on tissue and skin.

\section{Conclusion}

We showed that diagnostics based on $\mathrm{THz}$ time domain spectroscopy allows to make an integral estimation of the content of metabolites in blood plasma and distinguish blood plasma of healthy rats and rats with experimental diabetes of different degrees of severity. To increase diagnostic $\mathrm{THz}$ sensitivity we now develop polymer capillary waveguide sensor for such solutions. We expect more sensitivity for refraction changes of the outer media (solution) due to tens of $\mathrm{cm}$ interaction length.

The ATR amplitude of human palm skin increased when the glucose concentrations in blood rose above the normal level. The observed change of the spectrum is described with high degree of accuracy by the reduction in the ratio $\Delta \varepsilon_{1} / \tau_{1}$ in the Debye model of glucose aqueous solution.

Terahertz Pulsed Spectroscopy can be used as a promising diagnostic method of Diabetes Mellitus.

The work is supported by RFBR grant 16-52-00222.

\section{References}

1. Angeluts, A.A., Gapeyev, A.B., Esaulkov, M.N. et al, Study of terahertz-radiation-induced DNA damage in human blood leukocytes // Quantum Electron. 2014. V. 44, No. 3. P. 247 - 251.

2. Cherkasova, O.P., Nazarov, M.M., Berlovskaya, E.E. et al, Studying human and animal skin optical properties by terahertz time-domain spectroscopy // Bulletin of the Russian Academy of Sciences: Physics. 2016. V. 80. No.4. P. 479-483.

3. Angeluts, A.A., Balakin, A.V., Evdokimov M.G. et al., Characteristic responses of biological and nanoscale systems in the terahertz frequency range // Quantum Electronics. 2014. V. 44. No.7. P. $614-632$.

4.Cherkasova, O.P., Nazarov, M.M., Angeluts, A.A., Shkurinov, A.P. Analysis of blood plasma at terahertz frequencies // Optics and Spectroscopy. 2016. V. 120. No.1. P. 50-57.

5. Nazarov, M.M., Cherkasova, O.P., Shkurinov. A.P. Study of the dielectric function of aqueous solutions of glucose and albumin using pulsed THz spectroscopy // Quantum Electronics. 2016. V. 46. No.6. P. 488-495.

6. Cherkasova, O.P., Nazarov M.M., Smirnova, I.N., Angeluts, A.A., Shkurinov, A.P. Application of Time-Domain $\mathrm{THz}$ Spectroscopy for Studying Blood Plasma of Rats with Experimental Diabetes // Physics of Wave Phenomena. 2014. V. 22. No. 3. P. 185-188.

7. Cherkasova, O.P., Nazarov, M.M., Shkurinov A.P. Noninvasive blood glucose monitoring in the terahertz frequency range // Optical and Quantum Electronics. 2016. V. 48. No.3. 